\title{
Amelioration of intestinal and systemic sequelae of murine Campylobacter jejuni infection by probiotic VSL\#3 treatment
}

Ira Ekmekciu', Ulrike Fiebiger ${ }^{1}$, Kerstin Stingl$^{2}$, Stefan Bereswill ${ }^{1}$ and Markus M. Heimesaat ${ }^{1 *}$

\begin{abstract}
Background: The incidence of human Campylobacter jejuni infections is progressively increasing worldwide. Probiotic compounds might open up valuable tools to decrease pathogen burden and subsequent pro-inflammatory immune responses, but in vivo data are scarce.

Methods and results: Secondary abiotic mice generated by broad-spectrum antibiotic treatment were perorally challenged with the commercial probiotic compound VSL\#3 consisting of Streptococcus thermophilus, Bifidobacterium breve, Bifidobacterium longum, Bifidobacterium infantis, Lactobacillus acidophilus, Lactobacillus plantarum, Lactobacillus paracasei, and Lactobacillus delbrueckii ssp. bulgaricus) either 5 days before (i.e. prophylactic regimen) or after (i.e. therapeutic regimen) peroral C. jejuni strain 81-176 infection, and analyzed 3 weeks following the initial bacterial reassociation. Upon challenge, mice were colonized with the probiotic bacteria and/or C. jejuni at comparable intestinal loads, but co-colonization did not result in reduction of the pathogen burden. Remarkably, prophylactic as well as therapeutic VSL\#3 treatment of C. jejuni infected mice ameliorated intestinal apoptosis and pro-inflammatory immune responses as indicated by lower numbers of innate and adaptive immune cell populations in the murine colon upon probiotic prophylaxis or treatment and reduced colonic concentrations of pro-inflammatory mediators including IL-6 and MCP-1. Importantly, concentrations of anti-inflammatory mediators such as IL-10 were significantly elevated in the colon of probiotics treated mice as compared to untreated controls. Strikingly, prophylactic VSL\#3 treatment attenuated C. jejuni induced systemic pro-inflammatory responses as indicated by less TNF and IL-12p70 secretion in the spleen of VSL\#3 pre-treated as compared to non-treated mice.
\end{abstract}

Conclusion: Administration of probiotic formulations such as VSL\#3 might open up valuable strategies for prophylaxis and/or treatment of C. jejuni induced intestinal and systemic sequelae in vivo by the suppression of pro-inflammatory and induction of anti-inflammatory responses.

Keywords: Probiotic compound, VSL\#3, Secondary abiotic mice, Gnotobiotic mice, Bacterial in vivo competition, Pathogen-commensal bacteria-host interaction, Apoptosis, Innate and adaptive immune cells, Pro-inflammatory cytokines, Anti-inflammatory cytokines, Extra-intestinal and systemic sequelae of infection

\section{Background}

The enteric bacterial pathogen Campylobacter jejuni is regarded as a commensal within the intestinal tract of wild and domestic animals, but highly virulent in

\footnotetext{
*Correspondence: Markus.heimesaat@charite.de

${ }^{1}$ Department of Microbiology and Hygiene, Charité-University Medicine Berlin, CC5, Campus Benjamin Franklin, FEM, Garystr. 5, 14195 Berlin, Germany

Full list of author information is available at the end of the article
}

humans acquiring the pathogen usually by consumption of contaminated products derived from livestock animals or contaminated surface water via the peroral route [1-3]. Whereas Campylobacter infections are on the rise worldwide [4-6], patients present with gastroenteritis of varying degree ranging from mild malaise and watery diarrhea to severe ulcerative colitis with inflammatory, bloody diarrhea [7]. In the vast majority of cases, intestinal disease resolves spontaneously, whereas 
systemic post-infectious sequelae including peripheral neuropathies such as Guillain-Barré-syndrome, MillerFisher syndrome or reactive arthritis might develop with a latency of weeks to months [8-10]. Due to the lack of suitable experimental in vivo models of campylobacteriosis, our understanding of the molecular mechanisms underlying Campylobacter-host interactions has been hampered for a long time $[3,11]$. Conventionally colonized mice, for instance, are protected from $C$. jejuni infection due to the host specific microbiota composition exerting physiological colonization resistance $[3,12]$. Previous results from our own experiments revealed that modification of the murine intestinal microbiota facilitated C. jejuni infection [12,13]. Upon virtual eradication of the intestinal microbiota by broad-spectrum antibiotic treatment secondary abiotic mice became highly susceptible to $C$. jejuni colonization and exhibited key features of human campylobacteriosis such as apoptosis and inflammation in the colon [12]. Notably, colonization resistance was restored in secondary abiotic mice recolonized with a murine microbiota. Thus, both secondary abiotic mice and secondary abiotic animals re-colonized with a murine microbiota are well suited to unravel the triangular relationship between intestinal pathogens, bacteria and the host immune system in vivo [12, 14].

Given the importance of the distinct intestinal microbiota composition in rendering the vertebrate host resistant against enteric pathogens including $C$. jejuni, interest in the potential of "beneficial" modulations of the microbiota composition in humans as well as in livestock animals has arisen. One promising strategy is the application of probiotics, defined as live microorganisms which, when administered in adequate concentrations, bestow health benefits to the host [15]. There are numerous indications from both in vitro and in vivo studies pointing out the efficacy of probiotics in therapy and prevention of enteric infections. Strains of probiotic microorganisms such as Lactobacillus acidophilus, Lactobacillus casei, Lactobacillus rhamnosus, Lactobacillus gasseri, and Bifidobacterium lactis, for instance, have been shown to inhibit growth, metabolism and adhesion of enteropathogenic bacteria including C. jejuni, Salmonella, Shigella, enterotoxigenic Escherichia coli or Vibrio cholerae to intestinal cells [16-20]. Furthermore, effects of probiotics have been examined in clinical studies for a number of gastrointestinal diseases. For instance, randomized trials suggest that co-administration of VSL\#3, a probiotic compound consisting of eight different bacterial strains [21], or Saccharomyces boulardii [22] significantly decrease the incidence of antibiotics associated diarrhea (AAD). Moreover, episodes of infectious diarrhea in both adults and children can be shortened by the use of probiotics [23]. A meta-analysis of 74 experimental studies, 84 clinical trials and more than 10,000 patients revealed that probiotics were effective in the therapy and prevention of several gastrointestinal diseases including AAD, Clostridium difficile toxin induced acute enterocolitis (the most severe form of AAD), infectious diarrhea, pouchitis and irritable bowel syndrome, but not of travelers' diarrhea or necrotizing enterocolitis [24]. However, the underlying mechanisms of the probiotic effect are yet not fully understood. Proposed mechanisms of action include, for instance, modification of the intestinal microbiota [25], enhancement of colonization resistance [26] and intestinal barrier functions [27], as well as modulation of innate and adaptive immune functions [28].

In the present study, we examined the beneficial effects exerted by prophylactic and therapeutic treatment of $C$. jejuni infected mice with the probiotic compound VSL\#3. We addressed, whether peroral VSL\#3 application would lower intestinal pathogenic burden in the host, downregulate $C$. jejuni induced pro-inflammatory sequelae and/or conversely, up-regulate anti-inflammatory immune responses not only locally (i.e. in the intestinal tract), but also in extra-intestinal compartments including systemic compartments.

\section{Methods}

\section{Generation of secondary abiotic mice}

Female $\mathrm{C} 57 \mathrm{BL} / 6 \mathrm{j}$ mice were bred and maintained within the same specific pathogen free (SPF) unit in the Forschungseinrichtungen für Experimentelle Medizin (FEM, Charité-University Medicine Berlin). Secondary abiotic mice virtually lacking an intestinal microbiota were generated by broad-spectrum antibiotic treatment for 8 weeks as described previously [29]. In brief, 8-10 week old mice were transferred to sterile cages and treated with a quintuple broad-spectrum antibiotic cocktail consisting of ampicillin plus sulbactam (1 g/L; Ratiopharm, Ulm, Germany), vancomycin (500 mg/L; Cell Pharm, Hannover, Germany), ciprofloxacin (200 mg/L; Bayer Vital, Leverkusen, Germany), imipenem (250 mg/L; MSD, Haar, Germany) and metronidazole ( $1 \mathrm{~g} / \mathrm{L}$; Fresenius, Bad Homburg, Germany) via the drinking water ad libitum for 8 weeks. Absence of cultivable bacteria in feces samples (applying thioglycolate enrichment broths; Oxoid, Wesel, Germany) for at least three consecutive weeks was used as a quality control for the successful depletion of the gut microbiota [29].

\section{Probiotic treatment and C. jejuni infection of secondary abiotic mice}

Three days prior to bacterial re-colonization or infection experiments the quintuple antibiotic cocktail was withdrawn and replaced by autoclaved tap water. Mice 
were perorally infected with $10^{9}$ colony forming units (CFU) C. jejuni strain 81-176 in $0.3 \mathrm{~mL}$ sterile phosphate buffered saline (PBS) by gavage as described earlier [12]. For probiotic re-colonization, mice received a suspension of the commercial formulation VSL\#3 (probiotic food supplement; Manufacturer: SIIT S.r.l, Trezzano sul Naviglio, Italy; distributed by Actial Farmaceutica, Funchal, Madeira, Portugal) consisting of the following eight bacterial species: Streptococcus thermophilus, Bifidobacterium breve, Bifidobacterium longum, Bifidobacterium infantis, Lactobacillus acidophilus, Lactobacillus plantarum, Lactobacillus paracasei, and Lactobacillus delbrueckii ssp. bulgaricus). A total of $4.5 \times 10^{11}$ probiotic bacteria were dissolved in $50 \mathrm{~mL}$ sterile PBS. By gavaging $0.3 \mathrm{~mL}$ (either five days before or after C. jejuni infection), each mouse received $10^{9}$ viable probiotic bacteria as confirmed by cultural analyses of the suspensions. Mice infected either with the pathogen or re-colonized with the probiotic formulation alone as well as naive uninfected mice served as controls. Mice were continuously kept in a sterile environment (autoclaved food and drinking water) and were handled under strict aseptic conditions to prevent from contaminations.

\section{Sampling procedures}

Tissue samples from colon, mesenteric lymph nodes (MLN) and spleen were removed under sterile conditions. Colonic ex vivo biopsies were collected in parallel for microbiological and immunological analyses. Immunohistopathological changes were determined in colonic samples that had been immediately fixed in $5 \%$ formalin and embedded in paraffin. Sections $(5 \mu \mathrm{m})$ were stained with respective antibodies for in situ immunohistochemistry as described earlier [30].

\section{Quantitative analysis of C. jejuni or probiotic bacterial colonization}

Viable C. jejuni strain 81-176 were detected in feces or at time of necropsy in luminal samples taken from the colon, dissolved in sterile PBS and serial dilutions cultured on Karmali- and Columbia-Agar supplemented with 5\% sheep blood (Oxoid, Wesel, Germany) for two days at $37{ }^{\circ} \mathrm{C}$ under microaerobic conditions using CampyGen gas packs (Oxoid) as described earlier [12]. Probiotic bacteria of the formulation VSL\#3 were quantitated in serial dilutions streaked onto Columbia-Agar supplemented with 5\% sheep blood and Columbia-CNA Agar supplemented with colistin and nalidixic acid (both Oxoid) in parallel and incubated under aerobic (with 5\% $\mathrm{CO}_{2}$ ), microaerophilic (in jars using CampGen gas packs; Oxoid) and obligate anaerobic (in jars using Anaerogen gas packs; Oxoid) conditions for at least 2 days. Bacterial species were identified according to their typical morphological appearances and confirmed by $16 \mathrm{~S}$ rRNA based sequencing. The total probiotic bacterial loads in intestinal samples were assessed by the sum of identified CFU derived from the respective culture conditions. The detection limit of viable bacteria was $\approx 100$ CFU per $g$.

Immunohistochemical stainings of colonic ex vivo biopsies In situ immunohistochemical analysis of colonic paraffin sections was performed as described previously [12, 31, 32]. Primary antibodies against cleaved caspase-3 (Asp175, Cell Signaling, USA, 1:200), Ki67 (TEC3, Dako, Glostrup, Denmark, 1:100), CD3 (\#N1580, Dako, Denmark, dilution 1:10), FOXP3 (FJK-16s, eBioscience, San Diego, CA, USA, 1:100), B220 (eBioscience, 1:200) and F4/80 (\# 14-4801, clone BM8, eBioscience, 1:50) were used. The average numbers of positively stained cells within at least six high power fields (HPF, 400× magnification) were determined for each animal microscopically by an independent blinded investigator.

\section{Cytokine detection in culture supernatants of ex vivo biopsies taken from colon, mesenteric lymph nodes and spleen}

Colonic ex vivo biopsies were cut longitudinally and washed in PBS. MLN, spleen or strips of approximately $1 \mathrm{~cm}^{2}$ colonic tissue were placed in 24-flat-bottom well culture plates (Nunc, Wiesbaden, Germany) containing $500 \mu \mathrm{L}$ serum-free RPMI 1640 medium (Gibco, life technologies, Paisley, UK) supplemented with penicillin (100 $\mathrm{U} / \mathrm{mL})$ and streptomycin $(100 \mu \mathrm{g} / \mathrm{mL}$; PAA Laboratories, Pasching, Austria). After $18 \mathrm{~h}$ at $37{ }^{\circ} \mathrm{C}$, culture supernatants were tested for TNF, MCP-1, IL-6, IL-12p70, and IL-10 by the Mouse Inflammation Cytometric Bead Assay (CBA; BD Biosciences, Heidelberg, Germany) on a BD FACSCanto II flow cytometer (BD Biosciences).

\section{Statistical analysis}

Medians and levels of significance were determined using Mann-Whitney test (GraphPad Prism v5, La Jolla, CA, USA) as indicated. Two-sided probability (p) values $\leq 0.05$ were considered significant.

\section{Results}

Intestinal colonization densities in secondary abiotic mice following peroral re-colonization with probiotic bacteria and/or C. jejuni strain 81-176 infection

In the present study we investigated the potential of probiotic bacteria in the commercial formulation VSL\#3 to reduce pathogen burdens and to ameliorate pro-inflammatory immune responses upon $C$. jejuni infection in vivo. To address this, secondary abiotic mice were generated by broad-spectrum antibiotic treatment of conventionally reared mice. These mice were virtually lacking 
intestinal bacteria and hence, physiological colonization resistance was abrogated to assure stable intestinal probiotic bacterial colonization and/or $C$. jejuni infection $[11,12]$. Secondary abiotic mice were then perorally challenged with a probiotic suspension (i.e. VSL\#3) containing $10^{9}$ viable bacteria in total by gavage either 5 days before (i.e. prophylactic regimen) or after (i.e. therapeutic regimen) peroral $C$. jejuni strain 81-176 infection (with $10^{9} \mathrm{CFU}$ ) and compared to control mice that were either challenged by probiotic bacteria or C. jejuni alone. Uninfected, naive mice served as negative controls. In fact, probiotic bacteria as well as $C$. jejuni could stably colonize the murine intestinal tract, both with high median loads of approximately $10^{9} \mathrm{CFU}$ per gram feces, irrespective of the re-colonization regimen (n.s.; Fig. 1). Neither in the therapeutic nor prophylactic re-colonization group, however, probiotic bacteria were able to lower $C$. jejuni burden as indicated by comparably high pathogen loads in fecal samples over time, and the same was true the other way around (n.s.; Fig. 1c, d). If compared to $C$. jejuni mono-infected mice, however, fecal pathogen loads were approximately 0.25 orders of magnitude lower in mice of the probiotic treatment group at day 21 following C. jejuni infection ( $<<0.005$; Fig. 2).

Overall, mice could be stably re-associated with probiotic bacteria and/or C. jejuni, but co-colonization did not result in a biologically relevant reduction of either bacteria.

\section{Macroscopic and intestinal sequelae of $C$. jejuni infection and probiotic treatment}

Given that re-association with probiotic bacteria and/or C. jejuni strain 81-176 infection did not macroscopically (i.e. clinically) compromise secondary abiotic mice (not shown), we next investigated potential intestinal sequelae resulting from respective bacterial challenges. Given that apoptosis is a well-established marker for histopathological grading of intestinal inflammation and a key feature

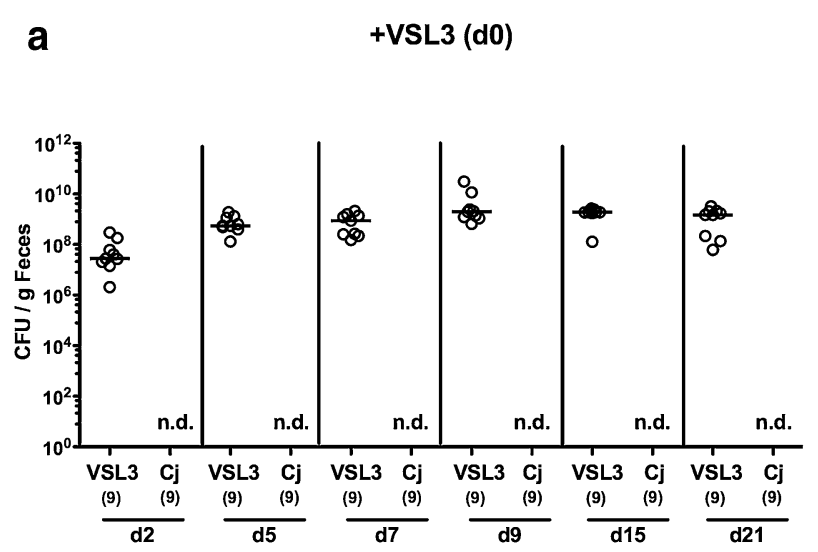

C

+VSL3 (d0) +C. jejuni 81-176 (d5)

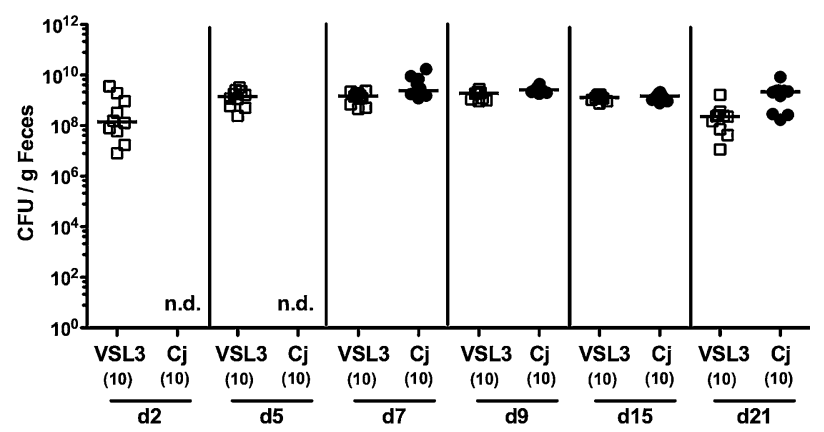

b

+C. jejuni 81-176 (d0)

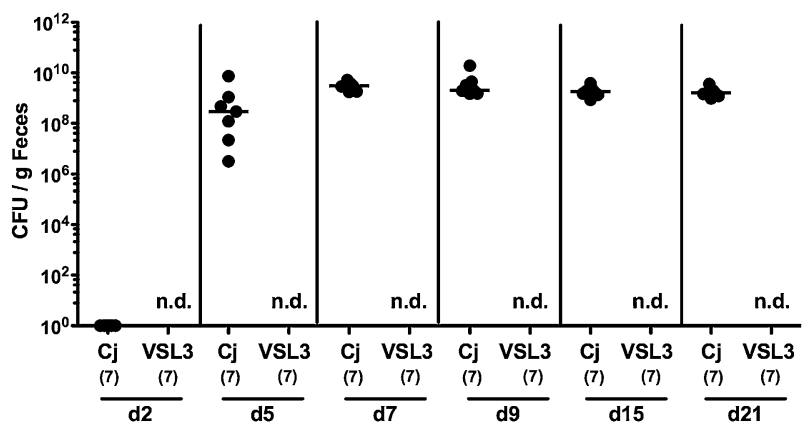

d +C. jejuni 81-176 (d0) +VSL3 (d5)

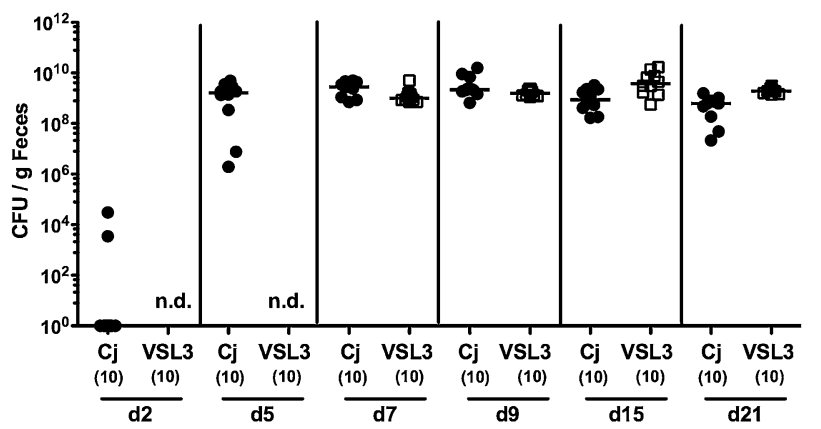

Fig. 1 Kinetic of intestinal probiotic bacterial and/or C. jejuni strain 81-176 loads in perorally associated secondary abiotic mice. Secondary abiotic mice were generated by broad-spectrum antibiotic treatment and perorally re-associated with a the probiotic compound VSL\#3 (white symbols) on day (d) 0, b C. jejuni strain 81-176 (Cj; black symbols) on d0, c VSL\#3 on day 0 and C. jejuni strain 81-176 on d5 or d C. jejuni strain 81-176 (d0) and VSL\#3 on d0 as described in "Methods". Bacterial colonization densities were assessed in fecal samples (CFU/g, colony forming units per gram) over time upon re-association as indicated by culture. Medians (black bars) and levels of significance ( $p$ value) determined by Mann-Whitney $U$ test are indicated. Numbers of analyzed mice are given in parentheses. Data were pooled from three independent experiments. N.d. not determined 


\section{Fecal Bacterial Loads (d21 p.i.)}

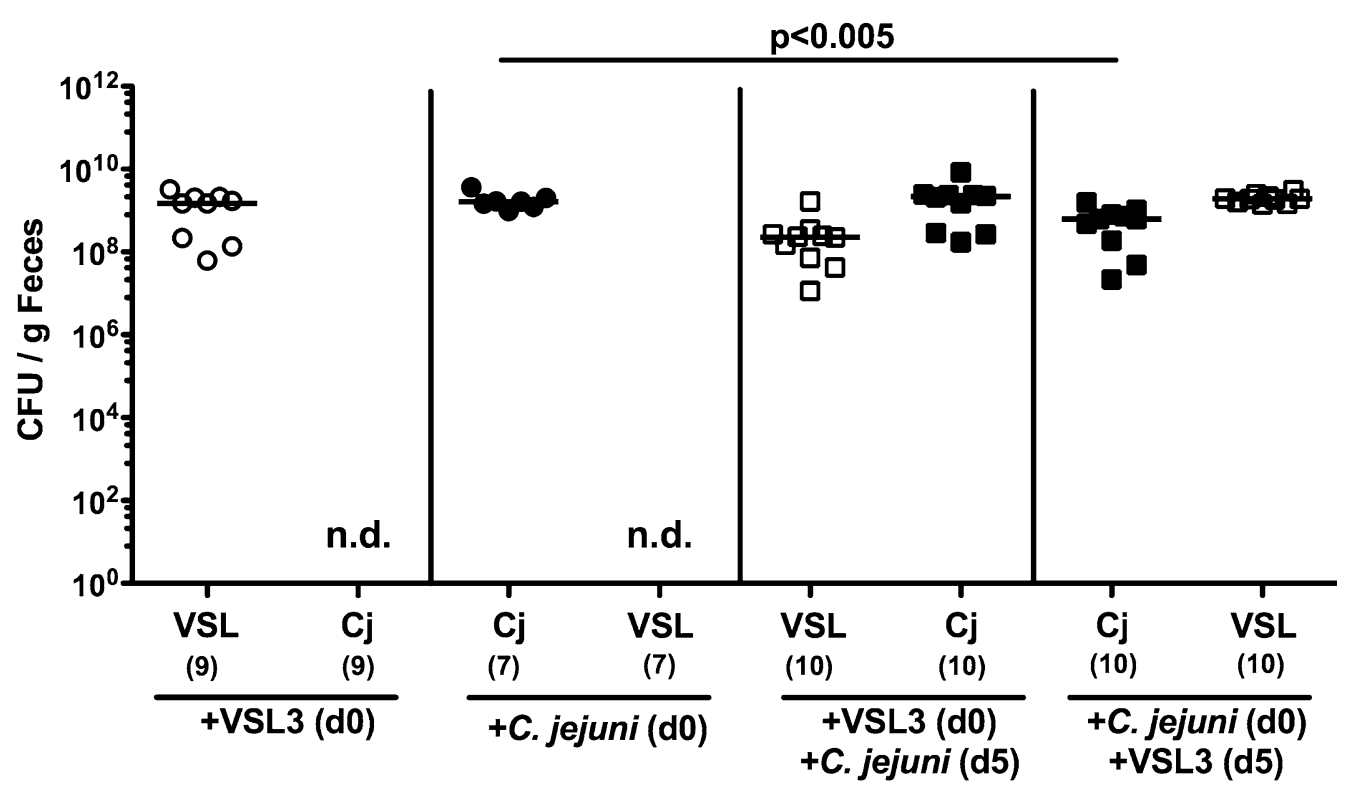

Fig. 2 Fecal bacterial loads in C. jejuni strain 81-176 infected and/or VSL\#3 associated secondary abiotic mice. Secondary abiotic mice were generated by broad-spectrum antibiotic treatment and perorally re-associated with the probiotic compound VSL\#3 (white symbo/s) on day (d) 0, C. jejuni strain 81-176 (Cj; black symbols) on day 0, VSL\#3 (day 0) and C. jejuni strain 81-176 (day 5) or C. jejuni strain 81-176 (day 0) and VSL\#3 (day 5) as described in "Methods". Bacterial colonization densities were assessed in fecal samples (CFU/g, colony forming units per gram) at day 21 upon initial re-association as indicated by culture. Medians (black bars) and level of significance ( $p$ value) determined by Mann-Whitney $U$ test are indicated. Numbers of analyzed mice are given in parentheses. Data were pooled from three independent experiments. N.d. not determined

of campylobacteriosis [12], we quantitatively assessed large intestinal epithelial caspase3 + cell numbers by in situ immunohistochemistry. Numbers of colonic apoptotic cells were higher in $C$. jejuni infected mice of either group as compared to naive or VSL\#3 mono-associated mice ( $\mathrm{p}<0.05-0.001$; Fig. 3a). These increases, however, were far less pronounced in VSL\#3 co-colonized mice of either regimen as indicated by approximately 50\% lower apoptotic cell numbers as compared to $C$. jejuni infected mice ( $p<0.005-0.001$; Fig. 3a). Notably, re-association of mice with the probiotic compound alone was not associated with colonic apoptosis (Fig. 3a). Given that Ki67 comprises a nuclear factor necessary for cellular proliferation [33], we additionally stained colonic paraffin sections with Ki67 antibodies to assess potential proliferative (and thus regenerative) measures of the large intestinal epithelium counteracting apoptosis. Bacterial or pathogenic mono- as well as co-association resulted in increases of $\mathrm{Ki} 67+$ colonic epithelial cell numbers ( $\mathrm{p}<0.001$; Fig. $3 \mathrm{~b}$ ) with a trend towards highest numbers in mice that were co-colonized with probiotic bacteria and $C$. jejuni (n.s. vs $C$. jejuni alone; $\mathrm{p}<0.05-0.005$ versus VSL\#3 alone; Fig. 3b). Hence, prophylactic as well as therapeutic challenge of $C$. jejuni infected mice with the probiotic compound VSL\#3 resulted in less pronounced large intestinal apoptotic responses.

\section{Intestinal and systemic pro- and anti-inflammatory responses upon probiotic treatment of $C$. jejuni infected mice}

Since recruitment of pro-inflammatory immune cells to the site of infection is a key feature of intestinal inflammation in the course of campylobacteriosis [12], we next quantitatively assessed distinct innate as well as adaptive immune cell subsets in large intestinal ex vivo biopsies, again applying in situ immunohistochemistry. Peroral C. jejuni infection, but not VSL\#3 re-colonization alone was associated with increases in colonic numbers of $\mathrm{T}$ and B lymphocytes, regulatory $\mathrm{T}$ cells (Treg) as well as macrophages and monocytes ( $p<0.001$; Fig. 4). These increases, however, were significantly less pronounced in with probiotics treated C. jejuni infected mice, irrespective whether VSL\#3 was applied prophylactically or therapeutically ( $\mathrm{p}<0.05-0.001$; Fig. 4).

We further measured pro- and anti-inflammatory cytokine concentrations in large intestinal ex vivo biopsies. Bacterial mono- as well as co-association were accompanied by increases in colonic pro-inflammatory 


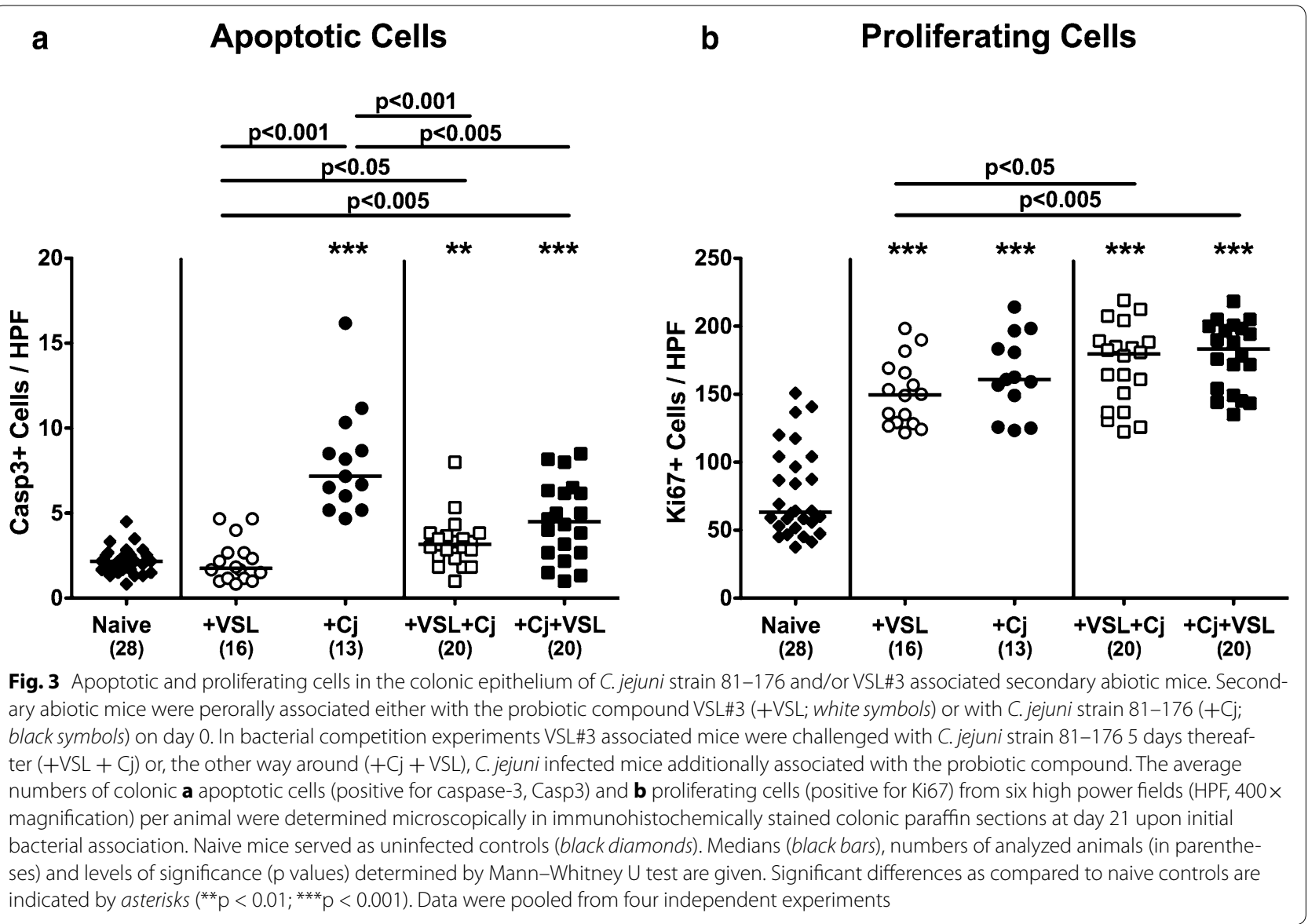

mediators including TNF, MCP-1, and IL-6 ( $\mathrm{p}<0.05-$ 0.001; Fig. 5a-c). C. jejuni induced increases in colonic MCP-1 and IL-6, but not increased TNF concentrations could be dampened by prophylactic probiotic treatment ( $\mathrm{p}<0.05$; Fig. 5a-c). Notably, IL-6 levels were also decreased in large intestines derived from $C$. jejuni infected mice of the therapeutic VSL\#3 cohort $(\mathrm{p}<0.05$; Fig. 5c) and did not differ from IL-6 concentrations measured in naive controls (n.s.; Fig. 5c). Notably, large intestinal concentrations of the anti-inflammatory cytokine IL-10 were increased upon mono- and co-association with the probiotic compound as compared to naive controls ( $\mathrm{p}<0.05-0.001$; Fig. 5d). Moreover, VSL\#3 application of either regimen resulted in elevated IL-10 concentrations in $C$. jejuni infected mice ( $<0.05$; Fig. $5 \mathrm{~d}$ ).

We next measured cytokine levels in another intestinal compartment. In MLN, concentrations of respective proand anti-inflammatory cytokines increased upon bacterial and/or pathogenic re-association ( $\mathrm{p}<0.05-0.001$; Fig. 6). Prophylactic probiotic treatment of $C$. jejuni infected mice, however, resulted in slightly lower IL-10 concentrations when compared to $C$. jejuni mono-associated mice ( $\mathrm{p}<0.05$; Fig. $6 \mathrm{~d})$.
We further assessed systemic cytokine responses upon bacterial and/or pathogenic challenges of secondary abiotic mice. C. jejuni induced increases in splenic TNF concentrations could be slightly lowered following probiotic pre-treatment ( $\mathrm{p}<0.05$; Fig. 7a), but not if probiotic treatment followed $C$. jejuni infection. In addition, mice of the prophylactic cohort exhibited lower IL-12p70 concentrations in their spleen as compared to $C$. jejuni infected animals ( $<<0.05$; Fig. $7 \mathrm{~b}$ ), whereas $C$. jejuni infection was associated with increased splenic IL-6 secretion ( $p<0.05-0.005$; Fig. $7 c$ ) that could neither be lowered by prophylactic nor therapeutic probiotic challenges. Notably, splenic IL-10 concentrations were unaffected upon bacterial re-colonization and/or pathogenic infection (n.s.; Fig. 7d).

Taken together, less colonic apoptosis upon probiotic co-administration in C. jejuni infected mice was accompanied by lower numbers of innate and adaptive immune cell populations in the large intestinal mucosa and lamina propria and less secretion of pro-inflammatory mediators, whereas anti-inflammatory IL-10 concentrations were increased in the colon upon prophylactic or therapeutic VSL\#3 treatment of C. jejuni infected 


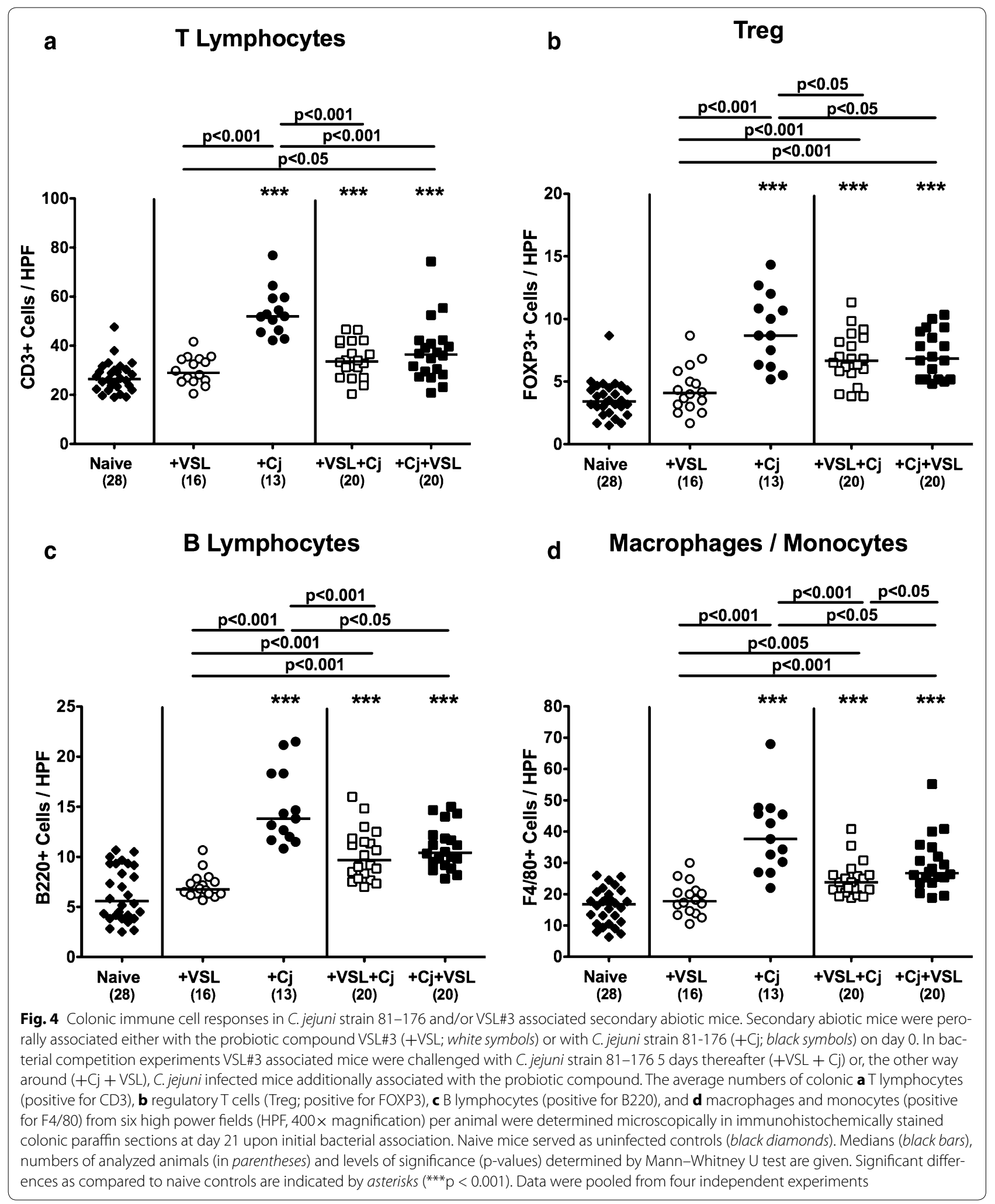




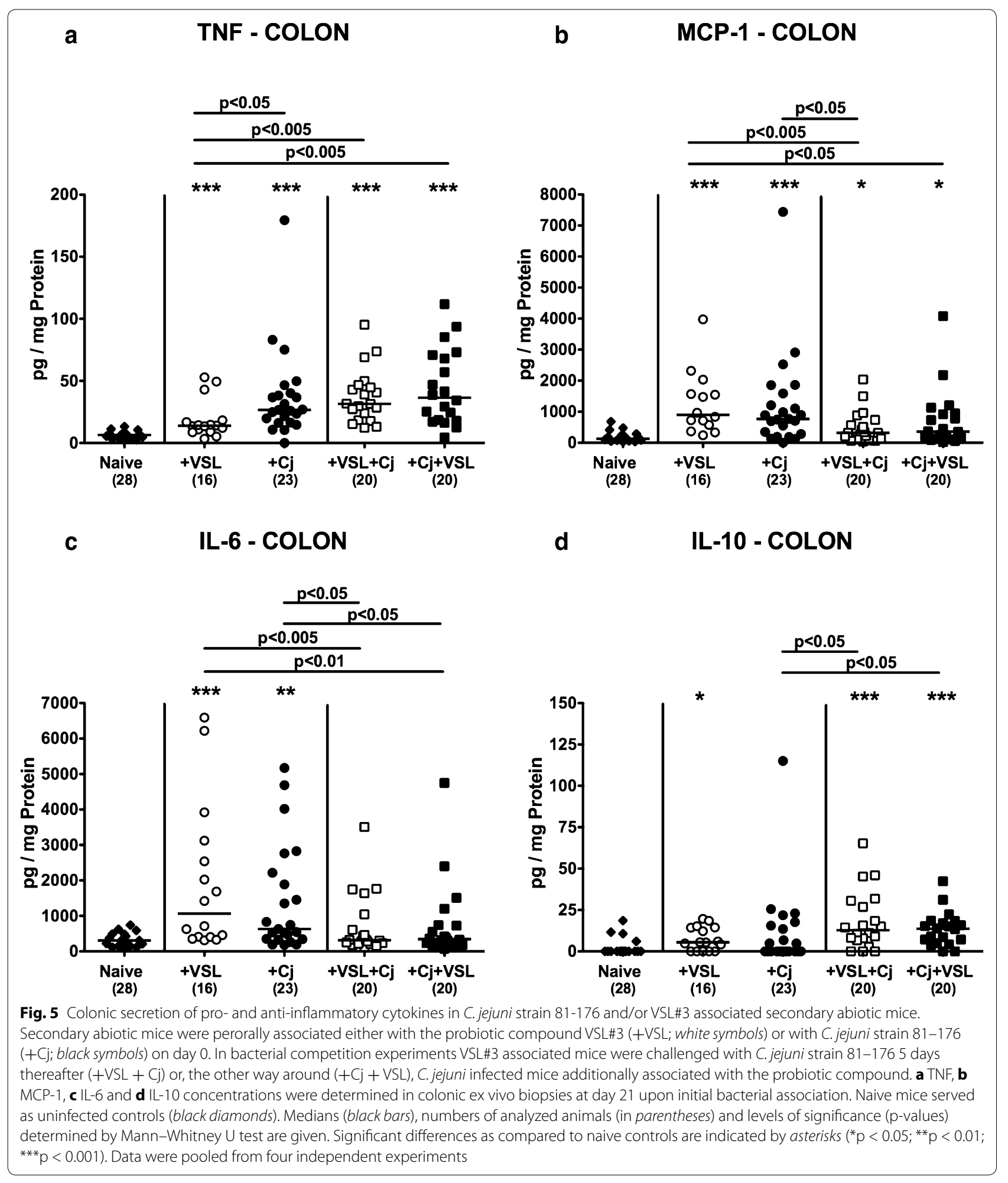

mice. Furthermore, prophylactic VSL\#3 challenge could dampen $C$. jejuni induced TNF responses in the systemic (i.e. splenic) compartment.

\section{Discussion}

Although C. jejuni are the most commonly reported bacterial etiological agents of diarrhea in developed 


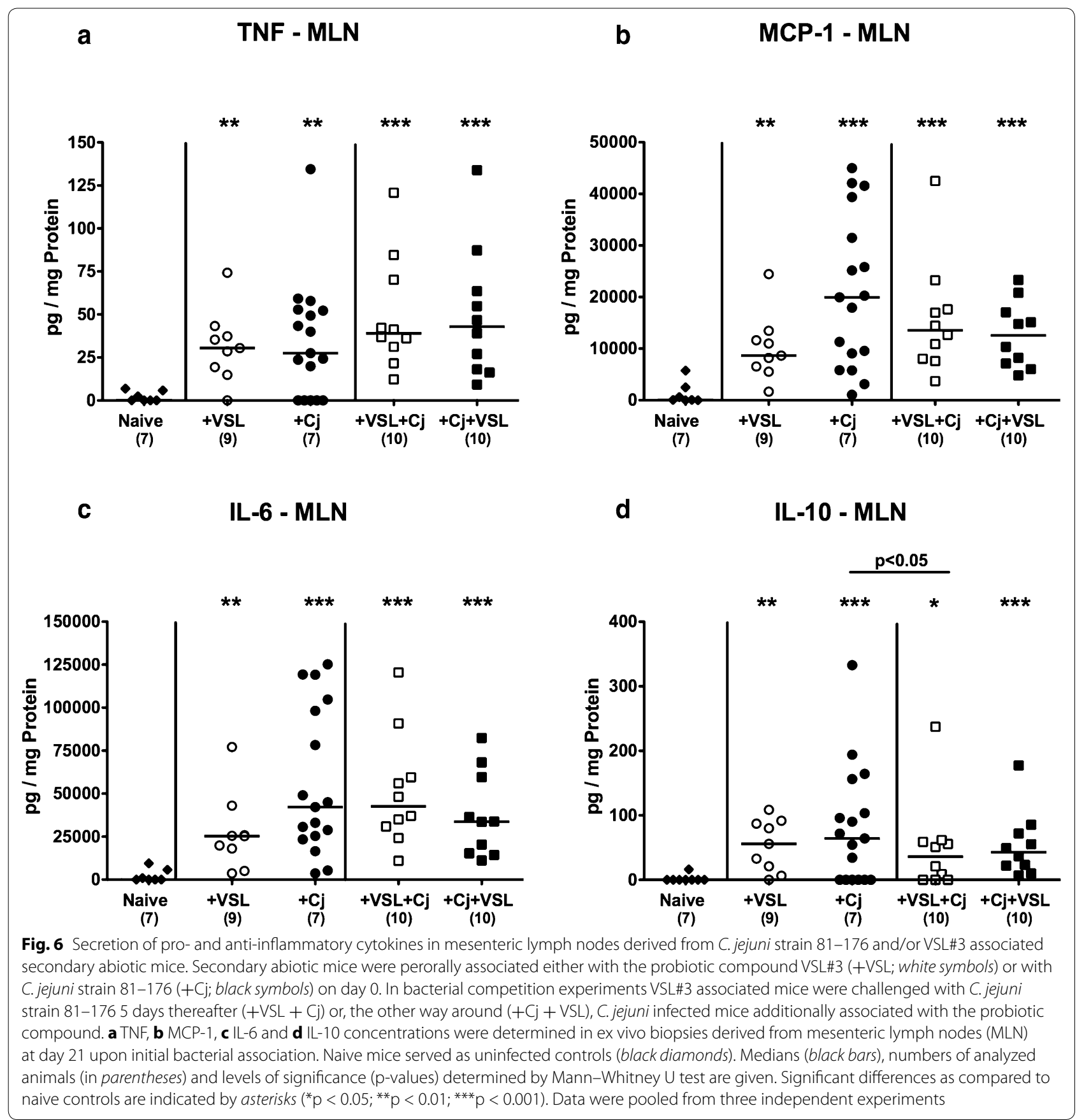

countries [3], in vivo data regarding the molecular mechanisms underlying pathogen-host interactions are still scarce, partly due to lack of suitable mouse models mimicking $C$. jejuni induced immunopathology in humans. We have previously shown that intestinal microbial depletion following antibiotic treatment of mice can overcome physiological colonization resistance against C. jejuni that is elicited by the murine host specific microbiota composition [12]. Apart from that, C. jejuni infected secondary abiotic mice exhibit immunopathological key features of human campylobacteriosis, thus providing a well-suited model to further unravel interactions between enteropathogens and the vertebrate host $[3,11,12]$. Given the importance of the distinct microbiota composition in disease susceptibility and progression, secondary modulation of the intestinal microbiota by application of probiotic compounds has arisen as an attractive preventive or therapeutic approach. In bacterial 


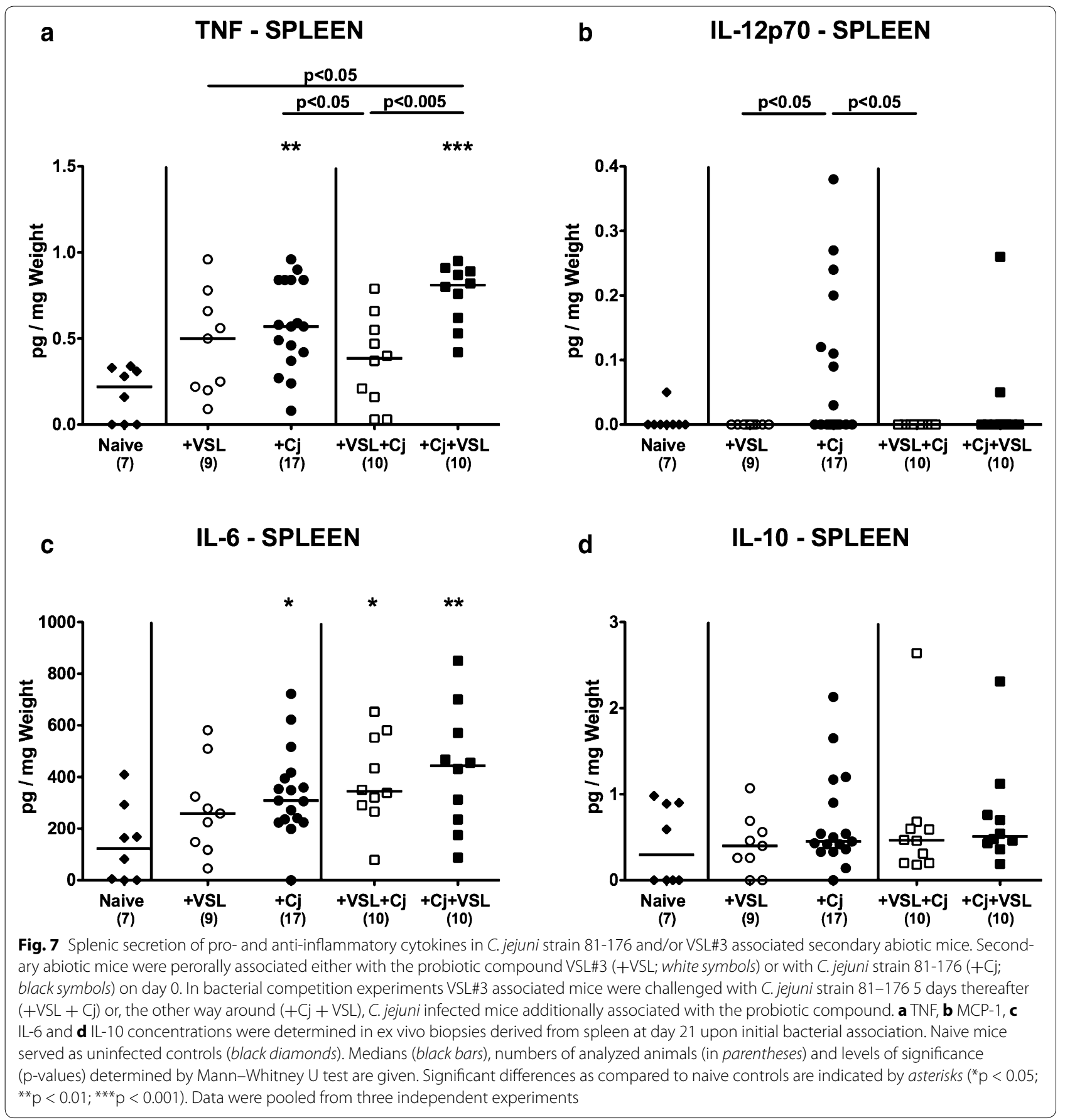

in vivo competition experiments applying our secondary abiotic mouse model, we here investigated changes in intestinal pathogen burden and host immune responses upon peroral C. jejuni infection and following pre- or post-treatment with the commercially available probiotic compound VSL\#3. Upon comparable and stable pathogenic as well as probiotic bacterial colonization of the intestinal tract, VSL\#3 could not sufficiently decrease intestinal $C$. jejuni loads in a biologically relevant manner within 3 weeks following initial bacterial challenge. Lowering the bacterial loads in livestock animals including poultry would be of great benefit in decreasing disease transmission rates to humans via the food chain, while immunomodulatory effects such as attenuation of intestinal inflammation would additionally result in less severe disease progression in the host. Our data are in 
contrast to a previous study applying isolator-raised ger$\mathrm{mfree}$ BALB/c mice that had been re-associated with a complex human microbiota and treated with a probiotic mix of five different Lactobacillus and three Bifidobacterium strains [26]. Following peroral infection with $C$. jejuni the authors observed a complete eradication of $C$. jejuni from the small and large intestines of with probiotics pre-challenged "humanized" mice [26]. One needs to take into consideration, that the observed differences in pathogen-eradicative properties might be due to differences in the used probiotic mixtures and could also be explained by different immunological features of the applied animal models. Due to the lacking contact to any bacterial ligands and subsequent absence of immunological differentiation and stimulation, germfree mice exhibit only poorly-developed intestinal lymphatic tissues [34, 35]. It is thus highly reasonable that the immunological repertoire in formerly isolator raised germfree mice substantially differs from the secondary abiotic mice applied here that had been born, raised and housed under conventional conditions. In addition, reconstitution of secondary abiotic mice with eight different probiotic strains (abundant in the VSL\#3 compound) might not be sufficient to reconstitute the complex physiological prerequisites for effective competition with C. jejuni for nutrients and niches. Instead, a well-orchestrated interplay of mucosal immunity and the intestinal intraluminal milieu determined by the concert of the complex microbiota plus beneficial probiotic strains might be required to successfully combat and/or prevent from enteropathogenic infection.

Whereas neither antibiotic treatment nor bacterial reassociation compromised mice clinically, we detected more pronounced apoptotic responses in colonic epithelia following $C$. jejuni infection as reported by us previously [12, 14, 30, 36-38]. Remarkably, C. jejuni induced apoptosis could be alleviated by both therapeutic and prophylactic VSL\#3 application. This is well in line with a former study demonstrating the capacity of VSL\#3 to attenuate epithelial apoptosis in a murine dextran sodium sulphate (DSS) induced colitis model [39]. Notably, less apoptosis was associated with more than three times increased numbers of colonic epithelial Ki67+ cells as compared to naive counterparts indicative for up-regulated regenerative properties upon therapeutic as well as prophylactic VSL\#3 treatment. Given that enhanced cell proliferative activity is essential in tissue repair and cell regeneration, and thus prevents from loss of epithelial integrity [40], this VSL\#3 induced measure might counteract and prevent from pathogen-induced apoptosis. This mechanism has already been proposed for other probiotic species including E. coli Nissle 1917 [27]. The anti-apoptotic properties exerted by VSL\#3 were further paralleled by a dampened $C$. jejuni induced recruitment of pro-inflammatory innate immune cell subsets including macrophages and monocytes as well as adaptive immune cell populations such as $\mathrm{T}$ lymphocytes, Treg and B lymphocytes into the large intestinal mucosa and lamina propria. A VSL\#3 mediated attenuated influx of pro-inflammatory immune cells into the colonic mucosa has already been shown in murine trinitrobenzene sulfonic acid (TNBS) induced colitis [41]. Intestinal and extra-intestinal cytokine analyses in our study further revealed that both therapeutic and prophylactic application of the probiotic compound resulted in increased colonic secretion of the anti-inflammatory key cytokine IL-10, whereas pro-inflammatory IL-6 concentrations were decreased in large intestines as compared to untreated $C$. jejuni infected mice. In addition, VSL\#3 prophylaxis resulted in decreased secretion of colonic MCP-1 upon C. jejuni infection. Most strikingly, antiinflammatory properties of VSL\#3 were not restricted to the intestinal tract, but could also be observed systemically, given that prophylactic VSL\#3 treatment attenuated C. jejuni induced TNF and IL-12p70 secretion in the spleen. At the first glance it appeared somewhat confusing in this context that both mono- as well as prophylactic probiotic bacterial co-colonization resulted in elevated levels of the pro-inflammatory cytokine TNF in colon and MLN. This result was, however, further supported by a former study demonstrating that VSL\#3 can in fact stimulate the intestinal epithelium to produce TNF in response, which interestingly resulted in improved epithelial barrier function and prevention of intestinal disease such as experimental ileitis [42]. Furthermore, observations that TNF deficient mice were more susceptible to acute DSS colitis have led to the concept that TNF might have protective functions in normal gut homeostasis and intestinal epithelial integrity [43]. Prophylactic VSL\#3 challenge dampened $C$. jejuni induced systemic TNF responses, however. While it may be physiologically important for the host to maintain a certain well-balanced level of local inflammation as a proper response to enteropathogens, avoidance of extra-intestinal and systemic inflammatory sequelae were pivotal for host health integrity. This further supports the dichotomic properties of cytokines depending on the respective (patho-)physiological context and cytokine milieu determining whether the same cytokine acts rather pro- or anti-inflammatory $[44,45]$. Our findings here further reinforce former data providing evidence for anti-inflammatory properties of VSL\#3. For instance, VSL\#3 has been shown to suppress MCP-1 production from human dendritic cells in vitro [46] and to down-regulate colonic MCP-1 mRNA expression also in vivo [47]. Moreover VSL\#3 application could ameliorate recurrent Th1-mediated TNBS colitis 
in mice by inducing IL-10 and IL-10-dependent regulatory T cells expressing TGF- $\beta$ [48]. The observed immunomodulatory effects of the probiotic compound might, at least in part, be attributed to the impact of probiotics on Toll-like receptor (TLR) expression [49]. We have previously reported that $C$. jejuni induced immunopathology depends on TLR-4- and TLR-9 signaling [12], whereas in a very recent study VSL\#3 was shown to down-regulate TLR-2, TLR-3, TLR-4, and TLR-9 expression in vitro [50]. Whether the observed beneficial effects can be attributed to single bacterial species out of the eight probiotic strains within the compound VSL\#3 or the mutualistic/synergistic interaction of distinct strains warrants further investigations.

\section{Conclusion}

Our data presented here provide evidence that though not sufficiently enforcing pathogenic clearance, therapeutic as well as prophylactic VSL\#3 application can induce antiinflammatory responses and limit not only intestinal, but also systemic pro-inflammatory sequelae of vertebrate $C$. jejuni infection. The probiotic compound VSL\#3 might therefore further open up promising tools for prophylaxis and/or treatment of $C$. jejuni induced sequelae.

\section{Abbreviations \\ AAD: antibiotics-associated diarrhea; CFU: colony forming units; DSS: dextran sodium sulphate; MLN: mesenteric lymph nodes; PBS: phosphate buffered saline; SPF: specific pathogen free; TNBS: trinitrobenzene sulfonic acid; Treg: regulatory $T$ cells.}

\section{Authors' contributions}

Conceived and designed the experiments: IE MMH SB. Performed the experiments: IE UF MMH. Analyzed the data: IE UF MMH. Critically discussed the paper: IE KS SB MMH. Wrote the paper: IE MMH. Co-edited the paper: UF KS SB. All authors read and approved the final manuscript.

\section{Author details}

${ }^{1}$ Department of Microbiology and Hygiene, Charité-University Medicine Berlin, CC5, Campus Benjamin Franklin, FEM, Garystr. 5, 14195 Berlin, Germany. ${ }^{2}$ Department of Biological Safety, Federal Institute for Risk Assessment (BfR), National Reference Laboratory for Campylobacter, Berlin, Germany.

\section{Acknowledgements}

We thank Michaela Wattrodt, Ursula Rüschendorf, Silvia Schulze, Alexandra Bittroff-Leben, Ines Puschendorf, Gernot Reifenberger, and the staff of the animal research facility at Charité-University Medicine Berlin for excellent technical assistance and animal breeding.

\section{Competing interests}

The authors declare that they have no competing interests.

\section{Availability of data and materials}

Please contact author for data requests.

\section{Ethics approval}

All animal experiments were conducted according to the European Guidelines for animal welfare (2010/63/EU) with approval of the commission for animal experiments headed by the "Landesamt für Gesundheit und Soziales" (LaGeSo, Berlin, registration number G0184/12). Animal welfare was monitored twice daily by assessment of clinical conditions. Mice did not display any clinical symptoms during the entire experiment. Twentyone days following the initial bacterial re-colonization mice were sacrifized by isofluran treatment (Abbott, Greifswald, Germany).

\section{Funding}

This work was supported by grants from the German Research Foundation (DFG) to IE (SFB633, Immuco), SB (SFB633, TP A7), UF (SFB633, TP B6), MMH (SFB633, TP B6), from the German Federal Ministry of Education and Research (BMBF) to SB (TP1.1), and from the German Institute of Risk Assessment to SB/ $\mathrm{MMH}$ and $\mathrm{KS}$ (1329-526).

The funders had no role in study design, data collection and analysis, decision to publish or preparation of the manuscript.

\section{Publisher's Note}

Springer Nature remains neutral with regard to jurisdictional claims in published maps and institutional affiliations.

Received: 3 March 2017 Accepted: 5 April 2017

Published online: 11 April 2017

\section{References}

1. Guerry P, Szymanski CM. Campylobacter sugars sticking out. Trends Microbiol. 2008;16(9):428-35.

2. Lane JA, Mehra RK, Carrington SD, Hickey RM. The food glycome: a source of protection against pathogen colonization in the gastrointestinal tract. Int J Food Microbiol. 2010;142(1-2):1-13.

3. Masanta WO, Heimesaat MM, Bereswill S, Tareen AM, Lugert R, Gross $U$, et al. Modification of intestinal microbiota and its consequences for innate immune response in the pathogenesis of campylobacteriosis. Clin Dev Immunol. 2013;2013:526860.

4. Young KT, Davis LM, Dirita VJ. Campylobacter jejuni: molecular biology and pathogenesis. Nat Rev Microbiol. 2007:5(9):665-79.

5. Nachamkin I, Shadomy SV, Moran AP, Cox N, Fitzgerald C, Ung H, et al. Anti-ganglioside antibody induction by swine (A/NJ/1976/H1N1) and other influenza vaccines: insights into vaccine-associated Guillain-Barre syndrome. J Infect Dis. 2008;198(2):226-33.

6. Dasti JI, Tareen AM, Lugert R, Zautner AE, Gross U. Campylobacter jejuni: a brief overview on pathogenicity-associated factors and disease-mediating mechanisms. Int J Med Microbiol. 2010;300(4):205-11.

7. Kist M, Bereswill S. Campylobacter jejuni. Contrib Microbiol. 2001;8:150-65.

8. Talukder RK, Sutradhar SR, Rahman KM, Uddin MJ, Akhter H. Guillian-Barre syndrome. Mymensingh Med J. 2011;20(4):748-56.

9. Wakerley BR, Uncini A, Yuki N. Guillain-Barre and Miller Fisher syndromes-new diagnostic classification. Nat Rev Neurol. 2014;10(9):537-44.

10. Backert S, Tegtmeyer N, Cróinín TÓ, Boehm M, Heimesaat MM. Chapter 1-human campylobacteriosis. In: Klein G, editor. Campylobacter-features, detection, and prevention of foodborne disease. London: Elsevier, Academic Press; 2017. p. 1-25.

11. Heimesaat MM, Bereswill S. Murine infection models for the investigation of Campylobacter jejuni-host interactions and pathogenicity. Berl Münch Tierärztl Wochenschr. 2015;128(3-4):98-103.

12. Bereswill S, Fischer A, Plickert R, Haag LM, Otto B, Kühl AA, et al. Novel murine infection models provide deep insights into the "menage a trois" of Campylobacter jejuni, microbiota and host innate immunity. PLoS ONE. 2011;6(6):e20953.

13. Haag LM, Fischer A, Otto B, Plickert R, Kühl AA, Göbel UB, et al. Intestinal microbiota shifts towards elevated commensal Escherichia coli loads abrogate colonization resistance against Campylobacter jejuni in mice. PLOS ONE. 2012;7(5):e35988.

14. Alutis ME, Grundmann U, Fischer A, Hagen U, Kühl AA, Göbel UB, et al. The Role of Gelatinases in Campylobacter Jejuni Infection of Gnotobiotic Mice. Eur J Microbiol Immunol (Bp). 2015;5(4):256-67.

15. FAO/WHO. Guidelines for the evaluation of probiotics in food. Joint FAO/ WHO Working Group Report on Drafting Guidelines for the Evaluation of Probiotics in Food; 2002. ftp://fao.org/es/esn/food/wgreport2.pdf. 
16. Bernet-Camard MF, Lievin V, Brassart D, Neeser JR, Servin AL, Hudault S. The human Lactobacillus acidophilus strain LA1 secretes a nonbacteriocin antibacterial substance(s) active in vitro and in vivo. Appl Environ Microbiol. 1997;63(7):2747-53.

17. Hudault S, Lievin V, Bernet-Camard MF, Servin AL. Antagonistic activity exerted in vitro and in vivo by Lactobacillus casei (strain GG) against Salmonella typhimurium C5 infection. Appl Environ Microbiol. 1997;63(2):513-8.

18. Gopal PK, Prasad J, Smart J, Gill HS. In vitro adherence properties of Lactobacillus rhamnosus DR20 and Bifidobacterium lactis DR10 strains and their antagonistic activity against an enterotoxigenic Escherichia coli. Int J Food Microbiol. 2001;67(3):207-16.

19. Fernandez MF, Boris S, Barbes C. Probiotic properties of human lactobacilli strains to be used in the gastrointestinal tract. J Appl Microbiol. 2003;94(3):449-55.

20. Lee YK, Puong KY, Ouwehand AC, Salminen S. Displacement of bacterial pathogens from mucus and Caco-2 cell surface by lactobacilli. J Med Microbiol. 2003;52(10):925-30.

21. Selinger CP, Bell A, Cairns A, Lockett M, Sebastian S, Haslam N. Probiotic VSL\#3 prevents antibiotic-associated diarrhoea in a double-blind, randomized, placebo-controlled clinical trial. J Hosp Infect. 2013;84(2):159-65.

22. Szajewska H, Mrukowicz J. Meta-analysis: non-pathogenic yeast Saccharomyces boulardii in the prevention of antibiotic-associated diarrhoea. Aliment Pharmacol Ther. 2005;22(5):365-72.

23. Allen SJ, Okoko B, Martinez E, Gregorio G, Dans LF. Probiotics for treating infectious diarrhoea. Cochrane Database Syst Rev. 2004;2:Cd003048.

24. Ritchie ML, RomanukTN. A meta-analysis of probiotic efficacy for gastrointestinal diseases. PLoS ONE. 2012;7(4):e34938.

25. Isolauri E, Sutas Y, Kankaanpaa P, Arvilommi H, Salminen S. Probiotics: effects on immunity. Am J Clin Nutr. 2001;73(2 Suppl):444s-50s.

26. Wagner RD, Johnson SJ, Kurniasih Rubin D. Probiotic bacteria are antagonistic to Salmonella enterica and Campylobacter jejuni and influence host lymphocyte responses in human microbiota-associated immunodeficient and immunocompetent mice. Mol Nutr Food Res. 2009:53(3):377-88.

27. Ukena SN, Singh A, Dringenberg U, Engelhardt R, Seidler U, Hansen W, et al. Probiotic Escherichia coli Nissle 1917 inhibits leaky gut by enhancing mucosal integrity. PLoS ONE. 2007;2(12):e1308.

28. Grabig A, Paclik D, Guzy C, Dankof A, Baumgart DC, Erckenbrecht J, et al. Escherichia coli strain nissle 1917 ameliorates experimental colitis via toll-like receptor 2- and toll-like receptor 4-dependent pathways. Infect Immun. 2006;74(7):4075-82. doi:10.1128/iai.01449-05.

29. Heimesaat MM, Bereswill S, Fischer A, Fuchs D, Struck D, Niebergall J, et al. Gram-negative bacteria aggravate murine small intestinal Th1-type immunopathology following oral infection with Toxoplasma gondii. J Immunol. 2006;177(12):8785-95.

30. Heimesaat MM, Lugert R, Fischer A, Alutis M, Kühl AA, Zautner AE, et al. Impact of Campylobacter jejuni cj0268c knockout mutation on intestinal colonization, translocation, and induction of immunopathology in gnotobiotic IL-10 deficient mice. PLoS ONE. 2014;9(2):e90148.

31. Heimesaat MM, Nogai A, Bereswill S, Plickert R, Fischer A, Loddenkemper C, et al. MyD88/TLR9 mediated immunopathology and gut microbiota dynamics in a novel murine model of intestinal graft-versus-host disease. Gut. 2010;59(8):1079-87.

32. Haag LM, Fischer A, Otto B, Plickert R, Kühl AA, Göbel UB, et al. Campylobacter jejuni induces acute enterocolitis in gnotobiotic IL-10-/- mice via Toll-like-receptor-2 and -4 signaling. PLoS ONE. 2012;7(7):e40761.

33. Scholzen T, Gerdes J. The Ki-67 protein: from the known and the unknown. J Cell Physiol. 2000;182(3):311-22.
34. Savidge TC, Smith MW, James PS, Aldred P. Salmonella-induced M-cell formation in germ-free mouse Peyer's patch tissue. Am J Pathol. 1991;139(1):177-84.

35. Shroff KE, Cebra JJ. Development of mucosal humoral immune responses in germ-free (GF) mice. Adv Exp Med Biol. 1995;371a:441-6.

36. Alutis ME, Grundmann U, Fischer A, Kühl AA, Bereswill S, Heimesaat MM. Selective gelatinase inhibition reduces apoptosis and pro-inflammatory immune cell responses in Campylobacter jejuni-infected gnotobiotic IL-10 deficient mice. Eur J Microbiol Immunol (Bp). 2014;4(4):213-22.

37. Bereswill S, Alutis ME, Grundmann U, Fischer A, Göbel UB, Heimesaat MM. Interleukin-18 Mediates Immune Responses to Campylobacter jejuni Infection in Gnotobiotic Mice. PLoS ONE. 2016;11(6):e0158020.

38. Gölz G, Alter T, Bereswill S, Heimesaat MM. The immunopathogenic potential of Arcobacter butzleri-lessons from a meta-analysis of murine infection studies. PLOS ONE. 2016;11(7):e0159685.

39. Mennigen R, Nolte K, Rijcken E, Utech M, Loeffler B, Senninger N, et al. Probiotic mixture VSL\#3 protects the epithelial barrier by maintaining tight junction protein expression and preventing apoptosis in a murine model of colitis. Am J Physiol Gastrointest Liver Physiol. 2009:296(5):G1140-9.

40. Potten CS, Booth C, Pritchard DM. The intestinal epithelial stem cell: the mucosal governor. Int J Exp Pathol. 1997;78(4):219-43.

41. Mariman R, Kremer B, van Erk M, Lagerweij T, Koning F, Nagelkerken L. Gene expression profiling identifies mechanisms of protection to recurrent trinitrobenzene sulfonic acid colitis mediated by probiotics. Inflamm Bowel Dis. 2012;18(8):1424-33.

42. Pagnini C, Saeed R, Bamias G, Arseneau KO, Pizarro TT, Cominelli F. Probiotics promote gut health through stimulation of epithelial innate immunity. Proc Natl Acad Sci USA. 2009;107(1):454-9.

43. Naito Y, Takagi T, Handa O, Ishikawa T, Nakagawa S, Yamaguchi T, et al. Enhanced intestinal inflammation induced by dextran sulfate sodium in tumor necrosis factor-alpha deficient mice. J Gastroenterol Hepatol. 2003;18(5):560-9.

44. Munoz M, Heimesaat MM, Danker K, Struck D, Lohmann U, Plickert R, et al. Interleukin (IL)-23 mediates Toxoplasma gondii-induced immunopathology in the gut via matrixmetalloproteinase-2 and IL-22 but independent of IL-17. J Exp Med. 2009;206(13):3047-59.

45. Heimesaat MM, Alter T, Bereswill S, Gölz G. Intestinal Expression of genes encoding inflammatory mediators and gelatinases during Arcobacter Butzleri infection of gnotobiotic II-10 deficient mice. Eur J Microbiol Immunol (Bp). 2016;6(1):56-66.

46. Mariman R, Tielen F, Koning F, Nagelkerken L. The probiotic mixture VSL\#3 dampens LPS-induced chemokine expression in human dendritic cells by inhibition of STAT-1 phosphorylation. PLOS ONE. 2014;9(12):e115676.

47. Bassaganya-Riera J, Viladomiu M, Pedragosa M, De Simone C, Carbo A, Shaykhutdinov R, et al. Probiotic bacteria produce conjugated linoleic acid locally in the gut that targets macrophage PPAR $y$ to suppress colitis. PLoS ONE. 2012;7(2):e31238.

48. Di Giacinto C, Marinaro M, Sanchez M, Strober W, Boirivant M. Probiotics ameliorate recurrent Th1-mediated murine colitis by inducing IL-10 and IL-10-dependent TGF- $\beta$-bearing regulatory cells. J Immunol. 2005; 174(6):3237-46.

49. de Kivit S, Tobin MC, Forsyth CB, Keshavarzian A, Landay AL. Regulation of intestinal immune responses through TLR Activation: implications for pro- and prebiotics. Front Immunol. 2014;5:60.

50. Manuzak JA, Hensley-McBain T, Zevin AS, Miller C, Cubas R, Agricola B, et al. Enhancement of microbiota in healthy macaques results in beneficial modulation of mucosal and systemic immune function. J Immunol. 2016;196(5):2401-9. 